\title{
Anti-LIF Monoclonal Antibody MSC-1
}

National Cancer Institute

\section{Source}

National Cancer Institute. Anti-LIF Monoclonal Antibody MSC-1. NCI Thesaurus. Code C156176.

A humanized immunoglobulin G1 (IgG1) monoclonal antibody against leukemia inhibitory factor (LIF), with potential immunomodulating and antineoplastic activities. Upon intravenous administration, monoclonal antibody MSC-1 binds to LIF and inhibits LIF signaling by blocking the recruitment of glycoprotein 130 (gp130) to the LIF-LIF receptor (LIFR)-gp130 signaling complex. This inhibits signal transducer and activator of transcription 3 (ST AT 3) signaling and inhibits tumor cell growth. In addition, the inhibition of LIF signaling abrogates the immunosuppressive tumor microenvironment (TME) by decreasing immunosuppressive M2 macrophages and allows for the activation of natural killer (NK) cells and cytotoxic T-lymphocytes (CT Ls) against tumor cells. LIF, a member of the interleukin-6 (IL-6) family of cytokines, is involved in many physiological and pathological processes and plays an important role in both creating the TME and promoting the activity of cancer-initiating cells (CICs). LIF is overexpressed in many tumor cell types and its expression correlates with poor prognosis. 